\title{
Preparation of novel polymer-metal oxide nanocomposites with nanophase separated hierarchical structure
}

\author{
K NAM ${ }^{1,2}$, Y TSUTSUMI ${ }^{1}$, C YOSHIKAWA ${ }^{3}$, Y TANAKA ${ }^{1}$, R FUKAYA $^{1}$, T KIMURA $^{1,2}$, \\ H KOBAYASHI ${ }^{4}$, T HANAWA ${ }^{1, *}$ and A KISHIDA ${ }^{1,2, *}$ \\ ${ }^{1}$ Institute of Biomaterials and Bioengineering, Tokyo Medical and Dental University, 2-3-10 Kanda-Surugadai, \\ Chiyoda-ku, Tokyo 101-0062, Japan \\ ${ }^{2}$ Japan Science and Technology Agency, CREST 5, Sanbancho, Chiyoda-ku, Tokyo 102-0075, Japan \\ ${ }^{3}$ International Centre for Materials Nanoarchitectonics (MANA), National Institute for Materials \\ Science (NIMS), Tsukuba, Ibaraki 305-0044, Japan \\ ${ }^{4}$ Biomaterials Centre, National Institute for Materials Science (NIMS), Tsukuba, Ibaraki 305-0044, Japan
}

MS received 7 April 2010; revised 16 January 2011

\begin{abstract}
This article deals with preparation of nanocomposite which comprised of nanophase separated structure of polymer chains and metal oxide. By grafting poly(hydroxyethyl methacrylate), poly(HEMA) on the surface of titanium which is covered by passive titanium oxide by atom transfer radical polymerization (ATRP) and executing anodic polarization, hierarchy nanophase separated structure with controlled thickness can be obtained. The titanium ions would be cationically charged and completely filled up the unoccupied binding sites of the polymer chains via electrochemical reaction, eventually covering the polymer chains with titanium oxide. However, this structure can be obtained when the anodic polarization is executed at initial applied voltage exceeding $10 \mathrm{~V}_{\mathrm{SCE}}$. The control of thickness is possible by controlling the initial applied voltage. These results prove that the conventional polymer can form composite structure with metal oxide without using fillers or special polymers designed for composite.
\end{abstract}

Keywords. Atom transfer radical polymerization; anodic polarization; nanocomposite.

\section{Introduction}

The main purpose of the preparation of the nanocomposite is to obtain the synergic effect of the polymer and the inorganic compound. Nanocomposites have a peculiar structure, i.e. a phase separated structure, with a nanoscale interface between the polymer matrix and the inorganic compound (nanophase separated structure). This phase separated structure plays a very important role in the production of a molecular-level synergic effect between the organic and inorganic compounds in nanocomposites (Paul and Robeson 2008). In general, nanocomposites with a phase separated structure in nanoscale can be prepared by four different methods: (i) embedding an inorganic moiety into the polymer, (ii) using interpenetrating polymer networks with chemical bonds, (iii) incorporating inorganic groups by bonding them to the polymer backbone and (iv) forming a dual inorganicorganic hybrid polymer (Kickelbick 2003). These structures can be created by a sol-gel process, core-shell composite, the electrodeposition of conductive polymers

*Author for correspondence

(hanawa.met@tmd.ac.jp; kishida.fm@tmd.ac.jp) such as polyaniline, exfoliated claybased nanocomposite, and the self-assembly or assembly of nano building blocks for the formation of mesophase structures (Sudheendra and Raju 1999; Sanchez et al 2001; Kickelbick 2003; Lahav et al 2006; Angelomé et al 2005; Ivanovici et al 2008).

Nanocomposites with nanophase separated structures prepared by conventional methods have a common feature. The type of polymers that can be used for their preparation is limited to organic metals such as polyaniline or organo-functional metal alkoxides (Wessling 1997; Kickelbick 2003). This is because the affinity between organic and inorganic molecules is low, and the modification of physical property may allow the preparation of nanocomposites using only polymers and metal oxides. So, the selection of the polymer for the composite preparation is limited. Additionally, a polymer matrix exists as a gel phase or a polymer aggregate. Hence, it is very important to homogenously disperse the metal oxide throughout the polymer matrix in order to form a homogeneous nanophase separated structure. Alternative method for the creation of the nanocomposite without using organic metals is to apply the nanofiller such as nanoparticles or nanotubes to produce exfoliated claybased nanocomposite. This method is good in the fact that conventional polymer can be used for the nanocom- 
posite production and reinforcement of the mechanical strength, but possesses poor miscibility, dispersion and interfacial strength (Yang et al 2006; Xu et al 2008).

This paper describes a method for preparation of polymer-metal oxide nanocomposites with a nanophase separated structure, using conventional polymer such as poly (hydroxyethyl methacrylate) and metal oxide such as titanium oxide without using inorganic nanofillers or organic metals to construct electrode for biosensor. In order to prepare polymer-metal oxide nanocomposites with a nanophase separated structure, the homogeneous dispersion of metal ions, an increase in the concentration of the metal oxide, and a reduction in the size of the polymer-metal ion oxide interface are very important since they essentially alter the physical property of the nanocomposite. The most appropriate way would be to construct a hierarchical structure of polymer and metal oxide. It may be possible to achieve homogeneous metal oxide distribution with a high concentration by creating a layer of metal oxide between the polymer chains consisting of monolithic and continuous phase of metal oxide structure. The interface of polymer and metal oxide can be reduced to nanoscale when the polymer layer is a simple stretched polymer chain with uniaxial orientation. The monolithic and continuous phase of metal oxide structure which may function as the route for the electrons is required between non-electroconductive polymers to create a hetero-electroconductive surface.

Several requirements should be carefully considered in order to obtain such structures: a stretched polymer chain with a uniaxial orientation should be prepared, the problem of affinity between polymers and metal oxides should be solved and the metal oxide should be placed appropriately between the polymer chains. We attempted to control the rotational movement and uniaxial orientation of polymer chains by grafting them, i.e. by grafting a so called polymer brush on the surface of a substrate. This method is useful for completely suppressing the movement of one chain terminal; various polymers can thus be adopted. However, if the density of the grafted polymer chains is $<0.0065$ chains $/ \mathrm{nm}^{2}$, the chain will collapse, leading to the adsorption of the polymer onto the surface of the substrate (Tsujii et al 2006). Moreover, height of the grafted polymer chains should be equal; otherwise, blobs may be formed among the chains (de Gennes et al 1980).

Atom transfer radical polymerization (ATRP) via surface initiation can solve this problem. This method is useful since a high density polymer brush with a uniform chain length and fully extended chain conformation can be prepared (Tsujii et al 2006; Yoshikawa et al 2006). For the preparation of metal oxides, we carried out anodic polarization of titanium; this technique is beneficial because it is simple and height of the metal oxide layer formed on the surface of the substrate can be controlled by controlling the applied voltage (Weatherall 1992).
Since titanium is covered with passive titanium oxide where the composition is mainly $\mathrm{TiO}_{2}$, when the titanium surface is treated with anodic polarization, $\mathrm{TiO}_{2}$ is expected to grow between the unoccupied binding sites of the polymer brush by electrochemical reaction; it would thus be possible to create a 'metal oxide mesh' in the polymer brush (figure 1). Using this, we prepared a polymer-metal oxide nanocomposite with a nanophase separated hierarchical structure by grafting a polymer brush on the surface of a titanium substrate and carrying out anodic polarization.

\section{Experimental}

\subsection{Grafting high density polymer brush on titanium surface}

A titanium plate was mirror-polished with $\mathrm{SiC}$ paper and $\mathrm{SiO}_{2}$ suspension. The plates were ultrasonically washed in acetone and ethyl alcohol for 15 min each. The surface of the titanium plate was characterized by XPS, and it showed normal surface characteristics. The titanium substrate was covered with a $\mathrm{TiO}_{2}$ layer having a thickness of a few nanometers.

Hydroxyethyl methacrylate (HEMA) was purified under the conditions proposed by Beers et al (1999), and it was sealed and refrigerated. 6-(2-bromo-2-isobutyloxy)hexyltriethoxysilane (BHE) was synthesized according to the method reported by Ohno et al (Beers et al 1999; Ohno et al 2005). $\mathrm{Cu}(\mathrm{I}) \mathrm{Br}$, bipyridine and ethyl 2-bromoisobutylate (EBIB) were purchased and used without further purification. All the solvents used in this study were dehydrated before use. The modification of the titanium surface with the initiator was carried out by silane coupling (Ohno et al 2005; Yoshikawa et al 2006). The titanium substrate was immersed in a tetrahydrofuran solution of ammonium water $(1 \mathrm{wt} \%)$ and BHE (1 wt $\%)$ for $12 \mathrm{~h}$ at room temperature to chemically deposit BHE on its surface. Subsequently, the titanium surface with BHE was rinsed with THF and methanol to obtain a BHE initiated titanium substrate.

$\mathrm{Cu}(\mathrm{I}) \mathrm{Br}(25 \mathrm{mM})$ and bipyridine $(63 \mathrm{mM})$ were mixed in methanol for $\sim 30 \mathrm{~min}$ to solubilize $\mathrm{Cu}(\mathrm{I}) \mathrm{Br}$ completely. A mixture of HEMA $(4.5 \mathrm{M})$ and free initiator EBIB $(22.5 \mathrm{mM})$ was added to the methanol solution and mixed for a short period of time. A certain amount of this solution was then poured into a glass tube in which the BHE initiated titanium substrate was placed. The tube was sealed tightly and placed in a shaking water bath. Polymerization was carried out for $90 \mathrm{~min}$ at $40^{\circ} \mathrm{C}$. After polymerization, the polymer solution was diluted in dimethylformaldehyde (DMF) solution for performing gel permeation chromatography (GPC) (CCP\&8020-series high-speed liquid chromatograph, Tosoh, Japan) measurements equipped with two Shodex gel column LF804 


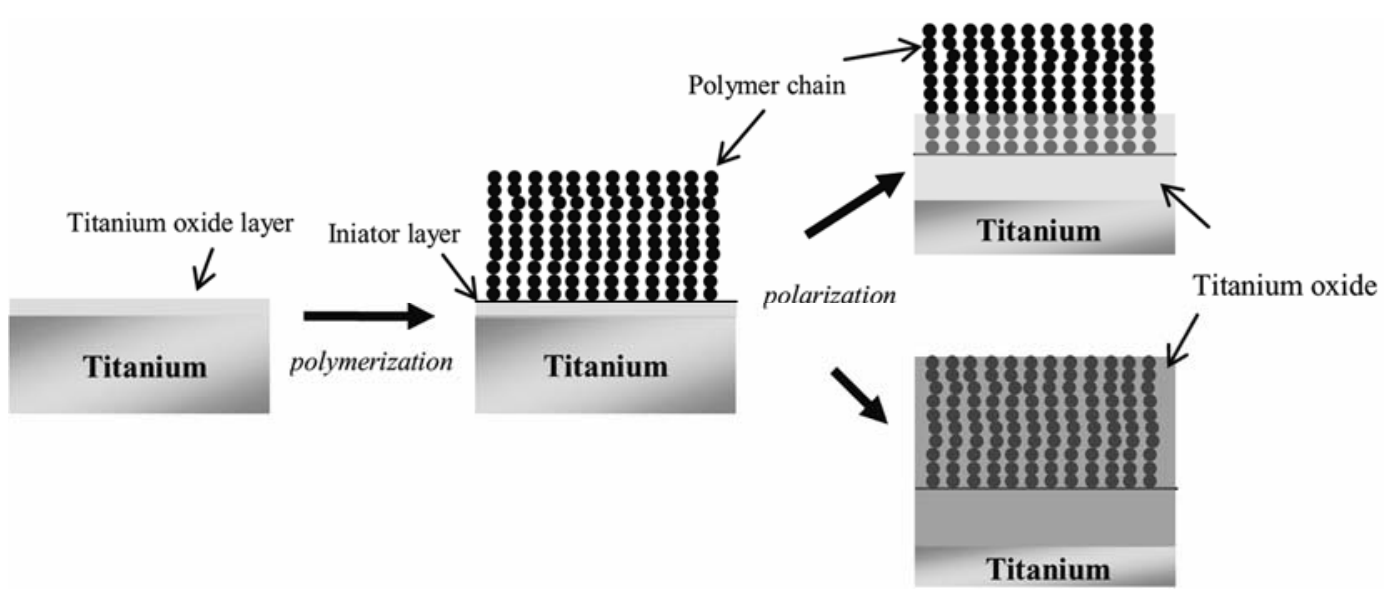

Figure 1. Preparation of polymer brush and polymer-metal oxide nanocomposite.

$(30 \times 080 \mathrm{~mm}$ : bead size $=6 \mu \mathrm{m}$, pore size $=20-3000 \AA)$ using refractive index. DMF was used as an eluent with a flow rate of $0.8 \mathrm{ml} / \mathrm{min}$, polyethylene glycol as the standard to calculate the polymer yield and polydispersity index (PDI). The thicknesses of the initiator layer and high density polymer brush grafted on the substrate were measured using an auto ellipsometer with a rotating analyzer (DVA-36L3, Mizojiri Optical Co., Japan). The polarizer angle was $45^{\circ}$ and the incident angle was $70^{\circ}$. The calculation of thickness of the brush layer was executed using the mathematical equation (Yoshikawa et al 2006):

$$
\sigma=L_{\rho} N_{\mathrm{A}} / M_{\mathrm{n}}
$$

where $L$ is the thickness of graft layer, $\rho$ the bulk density of poly(HEMA) $\left(1.15 \mathrm{~g} / \mathrm{cm}^{3}\right), N_{\mathrm{A}}$ the Avogadro number and $M_{\mathrm{n}}$ the number-average molecular weight.

All the procedures were carried out in an environment where the water and oxygen concentration in air was $<0.1 \mathrm{ppm}$. The surface of the titanium oxide, polymer brush and nanocomposite was observed with AFM (SPA300, Seiko Instruments Inc., Japan) using a silicon microcantilever (SI-AF01, Seiko Instruments Inc., Japan).

\subsection{Anodic polarization}

Anodic polarization was carried out by using a potentiostat (HA-501G, Hokuto Denko Corp., Japan). A saturated calomel electrode (SCE) and Pt electrode were used as reference and counter electrodes, respectively. The substrate was fixed in a polytetrafluoroethylene holder with an o-ring. The surface area of the substrate in contact with the electrolyte was $0.278 \mathrm{~cm}^{2}$. Details of the working electrode are described elsewhere (Tanaka et al 2007). After immersing the substrate into $0 \cdot 1 \mathrm{M} \mathrm{Na}_{2} \mathrm{SO}_{4}$ solution for $10 \mathrm{~min}$, anodic potentials of $10 \mathrm{~V}_{\mathrm{SCE}}$ were applied for $30 \mathrm{~s}$.

\subsection{Characterization}

In order to observe existence of polymer within the metal oxide, we chose nanocomposite prepared by anodic potentials of $10 \mathrm{~V}_{\mathrm{SCE}}$. X-ray photoelectron spectroscopic (XPS) and Auger electron spectroscopic (AES) studies were performed with an electron spectrometer (SSX-100, Surface Science Instruments, USA) and JAMP-7100, JEOL, Japan. All binding energies mentioned in this paper are relative to the Fermi level, and all spectra were excited with a monochromatized AlK $\alpha$ line $(1486 \cdot 61 \mathrm{eV})$. The photoelectron detector was placed at an angle of $35^{\circ}$ with respect to the substrate. The spectrometer was calibrated against the $\mathrm{Au} 4 f_{7 / 2}$ (binding energy: 84.07 eV) and $\mathrm{Au} 4 f_{5 / 2}(87.74 \mathrm{eV})$ levels of pure gold and $\mathrm{Cu} 2 p_{3 / 2}$ $(932.53 \mathrm{eV})$ and $\mathrm{Cu} 2 p_{1 / 2}(952.35 \mathrm{eV})$ levels and $\mathrm{Cu} \mathrm{Au}-$ ger L3M4,5M4,5 line (kinetic energy, $918.65 \mathrm{eV}$ ) of pure copper. The energy values were obtained from published data (Asami 1976). In order to estimate the photoelectron peak intensities, the background was subtracted from the measured spectrum according to Shirley's (1972) method.

\section{Results and discussion}

\subsection{Preparation of polymer brush on titanium substrate}

The composition of the passive titanium oxide surface before grafting the polymer brush was $26.4 \mathrm{~mol} \%$ of $\mathrm{Ti}$ and $73.6 \mathrm{~mol} \%$ of $\mathrm{O}$. The Ti $2 p$ spectrum showed four peaks $\left(\mathrm{Ti}^{0}, \mathrm{Ti}^{2+}, \mathrm{Ti}^{3+}\right.$ and $\left.\mathrm{Ti}^{4+}\right)$ and the $\mathrm{O} 1 \mathrm{~s}$ spectrum showed three peaks $\left(\mathrm{O}^{2-}: 56 \mathrm{~mol} \%, \mathrm{OH}^{-}: 29 \mathrm{~mol} \%\right.$ and $\left.\mathrm{H}_{2} \mathrm{O}: 11 \mathrm{~mol} \%\right)$. The calculated composition of surface of $\mathrm{TiO}_{2}$ substrate showed a high percentage of $\mathrm{TiO}_{2}$ and hydroxyl groups. The existence of the hydroxyl group on the surface of the material implies that the ATRP should be initiated by silane coupling. Hence, we 
Table 1. Characteristics of poly(HEMA) brush grafted on titanium substrate.

\begin{tabular}{|c|c|c|c|c|c|}
\hline & Thickness $^{\mathrm{a}}$ & Molecular weight ${ }^{b}$ & $M_{\mathrm{w}} / M_{\mathrm{n}}$ & $\sigma^{\varepsilon}$ & $d^{\mathrm{d}}$ \\
\hline Polymer brush & $16 \cdot 4$ & 20,300 & $1 \cdot 28$ & $0 \cdot 56$ & $1 \cdot 4$ \\
\hline
\end{tabular}

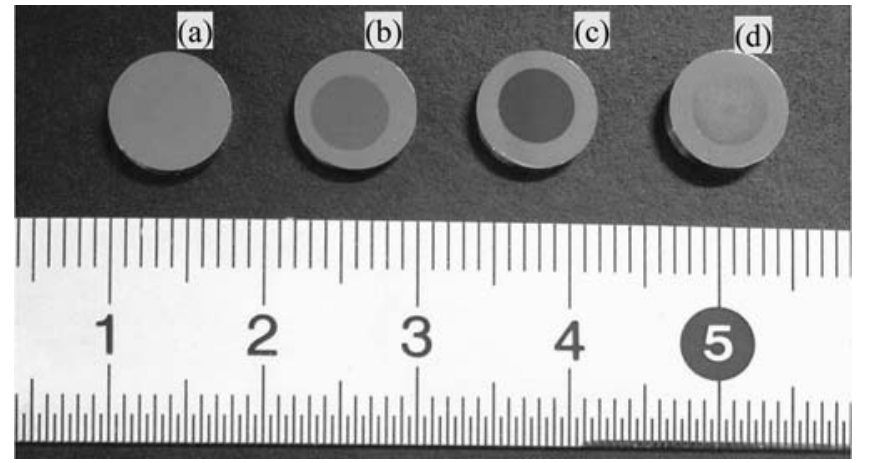

Figure 2. Image of (a) anodic polarized titanium substrate with polymer brush, $0 \mathrm{~V}_{\mathrm{SCE}}$, (b) anodic polarized titanium substrate with polymer brush, $3 \mathrm{~V}_{\mathrm{SCE}}$, (c) anodic polarized titanium substrate with polymer brush, $10 \mathrm{~V}_{\mathrm{SCE}}$ and (d) anodic polarized titanium substrate with polymer brush, $30 \mathrm{~V}_{\text {SCE }}$.

prepared a polymer brush by a 'surface initiation method' using (2-bromo-2-methyl)propionyloxyhexyltriethoxysilane (BHE) as the initiator (Ohno et al 2005; Yoshikawa et al 2006, 2010; Nam et al 2010). This highly reactive initiator reacts with hydroxyl groups via silane coupling.

Table 1 shows characteristics of the poly(hydroxyethyl methacrylate) (PHEMA) brush grafted on the passive titanium oxide surface. The mechanism of ATRP is simple; $\mathrm{Cu}(\mathrm{I})$ complex abstracts the halogen atom of the immobilized initiating dormant species or the grown dormant chain, giving a propagating radical. Then monomer units are added to it until it is recapped to be a dormant chain again. This cycle occurs repeatedly and randomly on the halogenated sites on the surface, which allows all graft chains to grow simultaneously in a controlled fashion (Tsujii et al 2006). In our case, density of the polymer brush calculated using (1) was 0.56 chains/ $\mathrm{nm}^{2}$ and its length was $\sim 16 \mathrm{~nm}$ when Mn was $\sim 2.0 \times$ $10^{4}$. The linear increase in the chain length according to the molecular weight of the poly(HEMA) was confirmed indicating that ATRP was successful (data not shown). According to Tsujii et al (2006), the conformation of a polymer brush on a substrate at this graft density is similar to that of a sphere that is randomly and irreversibly adsorbed on a flat surface. According to our calculation, the approximate distance between the polymer chains is $1.4 \mathrm{~nm}$ (de Ginnes 1980; Currie et al 2003) and the polydispersity index is 1.28 , implying that the polymer chain with uniform chain length has been prepared (Yoshikawa et al 2006, 2010; Nam et al 2010). The unoccupied binding sites of the polymer brush were available for the growth of $\mathrm{TiO}_{2}$, as shown in figure 1 . The polymer brush grafted on the passive titanium oxide surface was stable. Several researchers have reported that $\mathrm{Si}-\mathrm{O}-\mathrm{Ti}$ bonds are unstable; however, we could not find any evidence of the same (Xiao et al 1998; Dubrel et al 2006). Hence, we concluded that the high density poly(HEMA) brush grafted with on the passive titanium oxide surface with uniform chain length was successfully prepared and was chemically stable.

\subsection{Preparation of polymer-metal oxide nanocomposite}

From table 1, we concluded that the oxidized layer should be controlled to be at least $16 \mathrm{~nm}$ to create the hierarchy structure. Furthermore, it is thought that there is enough space for the titanium oxide to grow between the polymer chains, eventually covering the polymer brush layer. However, two questions must be answered in order to prepare a polymer-metal oxide nanocomposite: can anodic polarization occur, regardless of the existence of a dense polymer brush and can the metal oxide mesh be formed in between? Figure 2 shows images of titanium substrate after anodic polarization with the polymer brush. The interference colour of $\mathrm{TiO}_{2}$ was responsible for the change in colour, and it indicates change in thickness of the oxide layer (van Gils et al 2004). We have tested whether the change in colour according to the voltage is the same as bare titanium oxide surface. As shown in figure 3 , it can be seen clearly that the change in colour according to the voltage appears from $5 \mathrm{~V}_{\mathrm{SCE}}$, where the colour of titanium oxide was light gold. This is because increase in the titanium oxide layer depends on the initial applied voltage. The change of colour was exactly the same from that of bare titanium polarization. This indicates that the growth of $\mathrm{TiO}_{2}$ was not obstructed by the presence of the dense polymer brush. In other words, oxidation layer grew between the unoccupied binding sites of the polymer brush by electrochemical reaction and chemical reaction, contemporarily. The electrochemical reaction which induces the out-growth of the titanium oxide layer may be suppressed by the polymer chain. This would only induce the chemical reaction, which only makes the oxidization layer thicker inward. 

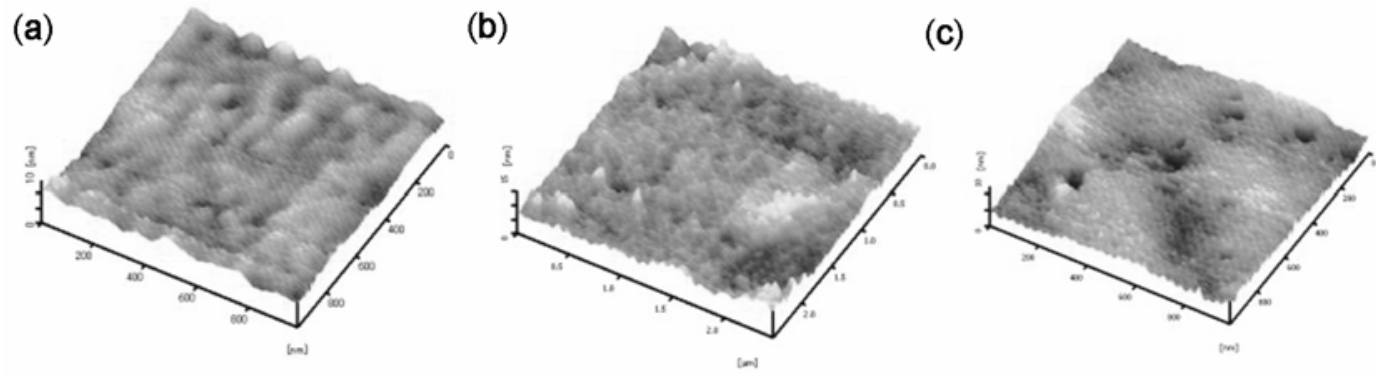

Figure 3. AFM images of (a) titanium substrate, (b) titanium substrate with polymer brush and (c) anodic polarized titanium substrate with polymer brush.
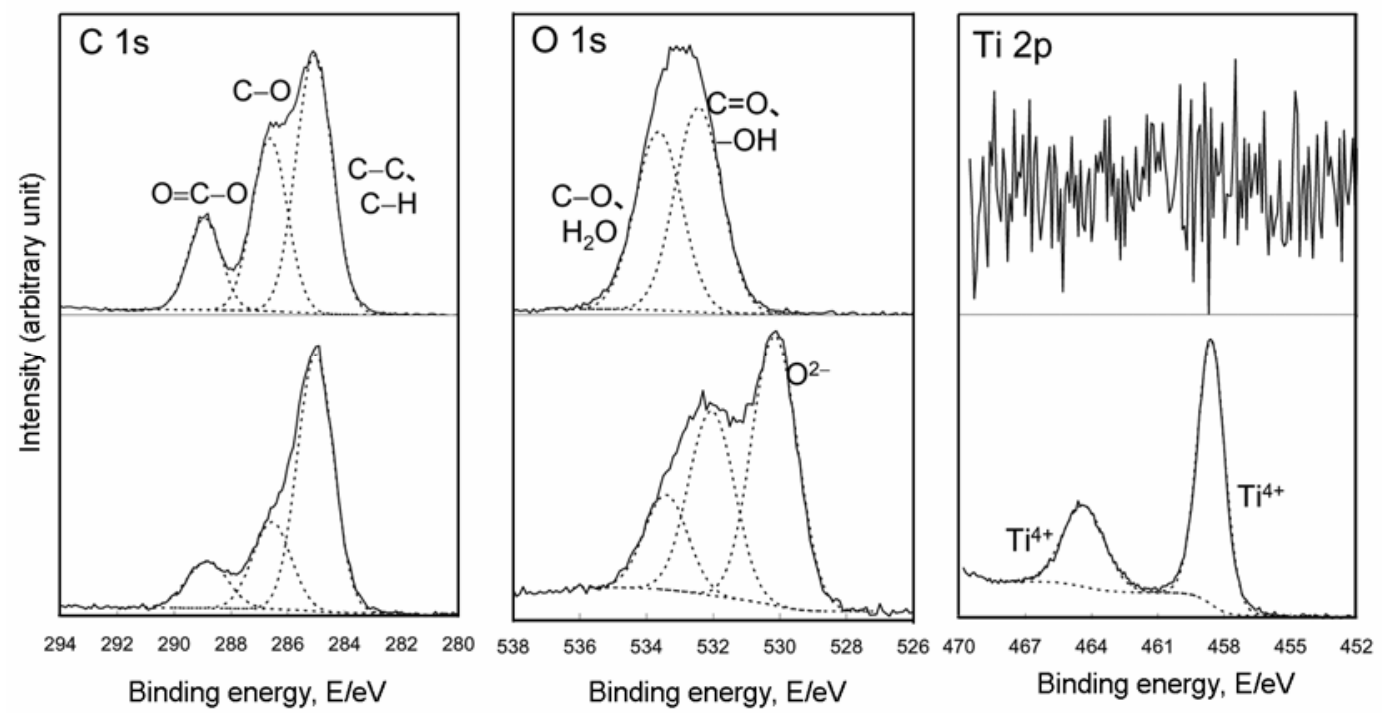

Figure 4. XPS results of titanium oxide with PHEMA brush layer. The upper images are the results of before anodic polarization and below images are the results of after anodic polarization.

\subsection{Surface of polymer-metal oxide nanocomposite}

The surface of the titanium, polymer brush and polarized titanium was observed with atomic force microscope (AFM). An AFM image of the substrate showed that it had a very smooth surface before and after polarization at $10 \mathrm{~V}_{\mathrm{SCE}}$ (figure 3). The surface of the polymer brush was very smooth because it contained polymer chains that had the same height. The surface after polarization was also very smooth, although it contained few holes. It is thought that the holes are produced by oxygen evolution occurring during the oxidation process. Nonetheless, surface of the titanium oxide layer was smooth and did not show any difference in height by more than $3 \mathrm{~nm}$. This implies that complete anodic polarization was achieved and the polymer chains did not interfere with the polarization process. However, we could not find any specific surface character for 1 or $5 \mathrm{~V}_{\mathrm{SCE}}$. Only flat surface was observed which did not provide us any information. So, it was required to confirm that the polymer chain exists after anodic polari- zation and the titanium oxide layer covers the polymer brush.

\subsection{Confirmation of nanocomposite structure}

To confirm the formation of the polymer-metal oxide composite, XPS and AES measurements were performed. Figure 3 shows XPS spectra for C $1 s, \mathrm{O} 1 s$ and Ti $2 p$ binding energies from outermost surface area on the nanometric scale $\left(10 \mathrm{~V}_{\mathrm{SCE}}\right)$. The presence of a thick and dense PHEMA brush on the titanium substrate before anodic polarization was confirmed (figure 4, upper images), because there was no peak corresponding to $\mathrm{TiO}_{2}$ in the $\mathrm{Ti} 2 p$ spectrum before the polymer brush was grafted. On the other hand, spectrum of the polarized substrate showed strong peaks in the $\mathrm{Ti} 2 p$ region; $\mathrm{Ti}^{4+}$ was determined as the binding state from the binding energy of $458 \mathrm{eV}$ (figure 4, below images). The spectrum of this substrate also showed a new peak due to the $\mathrm{O}^{2-}$ binding state at $529 \mathrm{eV}$ in the $\mathrm{O} 1 s$ region. Thus, the 

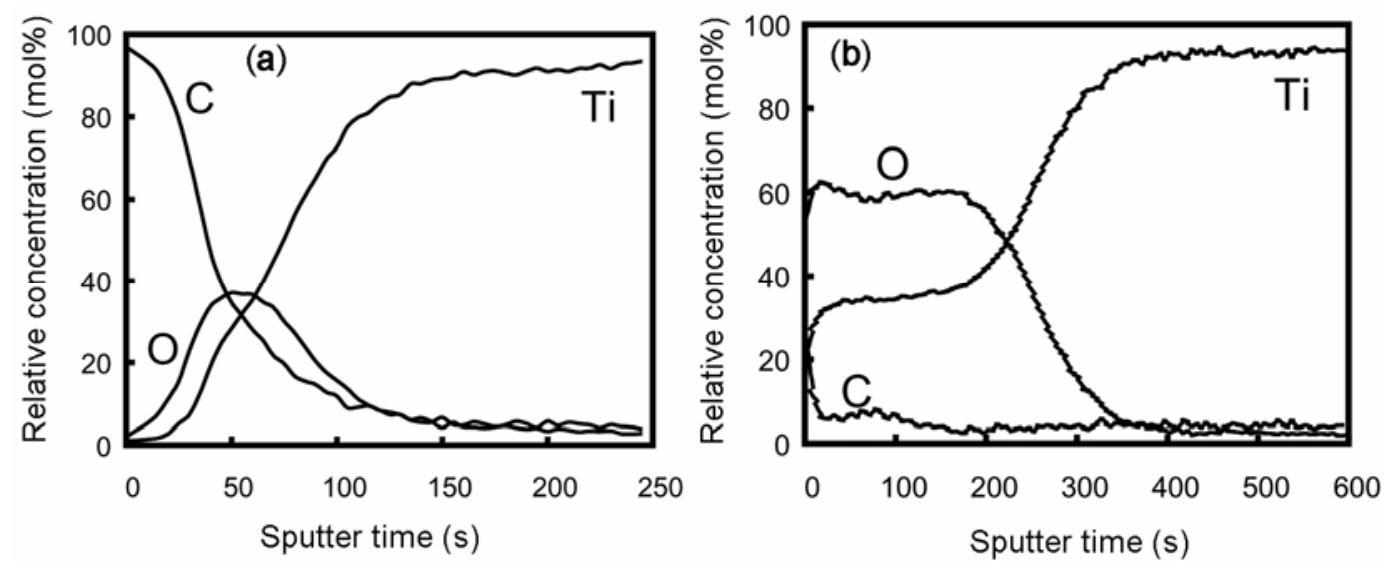

Figure 5. AES results of titanium substrate with PHEMA brush layer (a) before and (b) after anodic polarization.
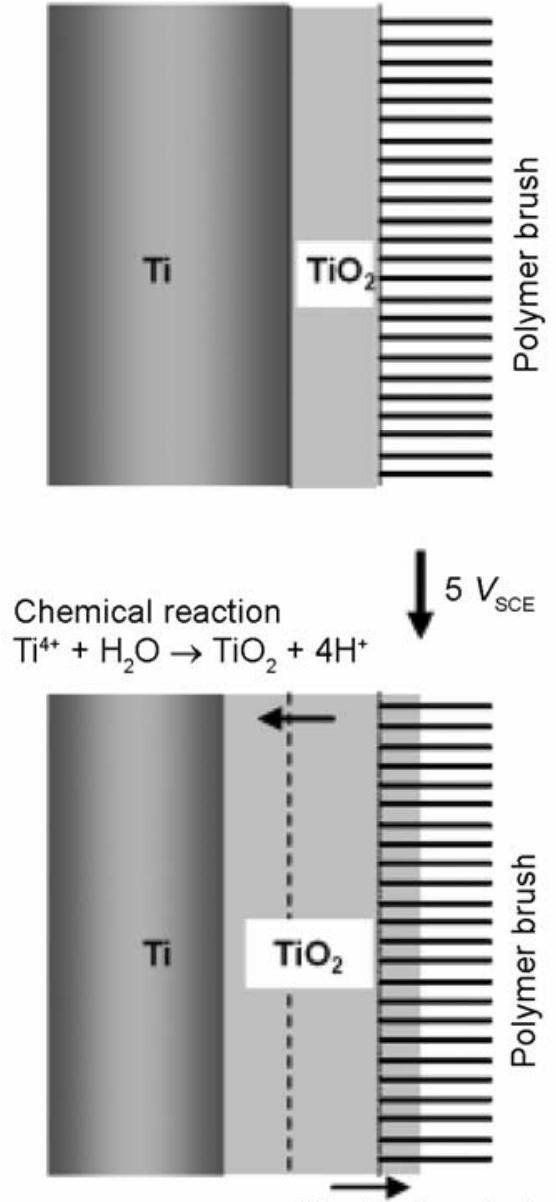

(a) Electrochemical reaction $\mathrm{Ti} \rightarrow \mathrm{Ti}^{4+}+4 \mathrm{e}^{-}$
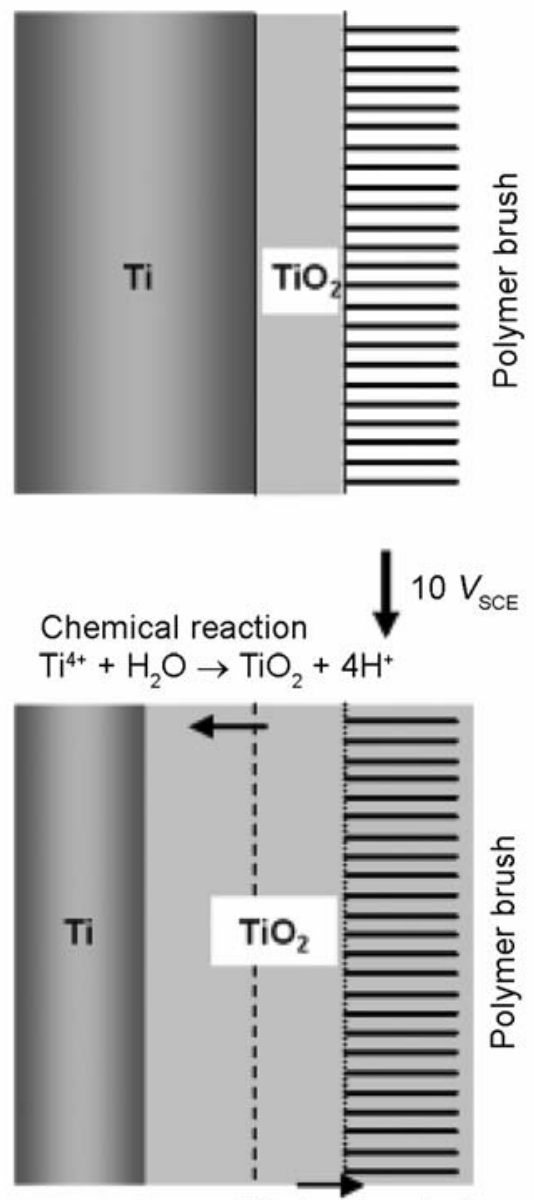

(b) Electrochemical reaction $\mathrm{Ti} \rightarrow \mathrm{Ti}^{4+}+4 \mathrm{e}^{-}$

Figure 6. Schematic image of electrochemical and chemical reaction forming titanium oxide layer by anodic polarization via (a) $5 \mathrm{~V}_{\mathrm{SCE}}$ and (b) $10 \mathrm{~V}_{\mathrm{SCE}}$.

surface of the anodic polarized substrate was almost covered with $\mathrm{TiO}_{2}$. These results indicate two important facts: the polymer brush is stable on the titanium surface in the aqueous solution and remained stable during the 
oxidation process, and the growth of the metal oxide by anodic polarization proceeded, covering the whole polymer brush. On the other hand, XPS result for $5 \mathrm{~V}_{\mathrm{SCE}}$ was exactly the same as the one with titanium substrate before grafting of the polymer chains (figure not shown), implying that the hierarchy structure of polymer-metal oxide was not formed or has less than $6 \mathrm{~nm}$ of the metal oxide formed by electrochemical reaction.

These results were further confirmed by performing an AES measurement for anodic polarization with $10 \mathrm{~V}_{\mathrm{SCE}}$ (figure 5). An AES depth profile measured before polarization showed three regions: a C-rich layer (PHEMA), an O-rich layer $\left(\mathrm{TiO}_{2}\right)$ and a Ti-rich layer (Ti metal). The surface was covered with carbon species, and this result agreed well with the result of XPS characterization. On the other hand, the polarized substrate showed a thick $\mathrm{TiO}_{2}$ layer. It should be noted that the left half (outer side) of the $\mathrm{TiO}_{2}$ layer also contained a certain amount of $\mathrm{C}$. The depth of this $\mathrm{C}-\mathrm{TiO}_{2}$ mixed layer was estimated to be about $20 \mathrm{~nm}$ with the sputter rate of a $\mathrm{SiO}_{2} / \mathrm{Si}$ standard sample, and this value corresponded to the height of the polymer brush. In other words, the upright polymer brush was covered and mixed with the metal oxide by anodic polarization. Hence, AFM result (figure 3) indicates that the polymer brush is located inside the $\mathrm{TiO}_{2}$ layer and the surface of the nanophase separated polymer-metal oxide nanocomposite is very smooth.

XPS and AES results are direct evidences of the presence of a nanophase separated polymer-metal oxide nanocomposite. However, the hierarchy structure can only be formed when the initial applied voltage is higher than $10 \mathrm{~V}_{\mathrm{SCE}}$. That is, the total thickness is required to be at least $30 \mathrm{~nm}$ to cover up the polymer brush with the metal oxide layer. Figure 6 shows schematic image of how the titanium oxide is formed according to the different initial applied voltages. The metal oxide layer which covers the polymer brush is electrochemical reaction. From this, it can be postulated that the balance between the in-growing oxidation layer by chemical reaction and out-growing oxidation layer by electrochemical reaction is very important. This is because length of the polymer brush is $\sim 16 \mathrm{~nm}$, and at least $16 \mathrm{~nm}$ is required to cover the whole polymer brush to from nanophase separated structure (table 1). In the case of low anodic polarization, the electrochemical reaction could not produce enough thickness to cover the polymer chain (figure 6). However, it is impossible to distinguish from the in-growing oxidation layer and out-growing oxidation layer and could not find any proof for this. It can be only assumed that the low initial voltage application have caused thicker formation of the in-growing oxidation layer by chemical reaction. So a comprehensive study on controlling thickness of the metal oxide by electrochemical reaction and chemical reaction is required.

\section{Conclusions}

We showed that the preparation method used in this study, which is a combination of simple and well known techniques, can be used to successfully produce novel and promising materials. We have obtained three important results. Firstly, contrary to reports on the instability of $\mathrm{Si}-\mathrm{O}-\mathrm{Ti}$ bonds, we found that polymer chains grafted on a $\mathrm{TiO}_{2}$ surface are stable. Secondly, the application of an appropriate voltage $\left(10 \mathrm{~V}_{\mathrm{SCE}}\right)$ to a titanium substrate with a polymer brush leads to the growth of $\mathrm{TiO}_{2}$ that completely fills up the unoccupied binding sites of the polymer chains, eventually covering the polymer layer with metal oxide. Finally, the polymer need not necessarily be compatible with the metal oxide. The key requirements for polymer-metal oxide nanocomposite preparation are as follows: the surface should have a relevant functional group that can react with the initiator, the polymer brush should be dense and have a homogenous chain length and an appropriate anodic polarization voltage should be applied for the growth of the metal oxide.

We are planning to adjust the thickness of the metal oxide layer to produce a nanoheterogenous surface consisting of organic and inorganic phases. An extensive study on this may lead to the establishment of a preparation method for a new type of surface with a hierarchical structure. Furthermore, it would be possible for us to construct an electrode for biosensor, applying this structure using other metals which is much suitable for electrode.

\section{Acknowledgements}

This work was partly supported by Core Research for Evolutional Science and Technology (CREST) of the Japan Science and Technology Agency (JST). The authors would like to acknowledge Prof. Y Tsujii, Kyoto University, Japan, for his advice on ATRP method.

\section{References}

Angelomé P C et al 2005 J. Mater. Chem. 153903

Asami K 1976 J. Electron Spectrosc. 9469

Beers K et al 1999 Macromolecules 325772

Currie E P K et al 2003 Adv. Colloid Interf. Sci. 100-102 205

de Gennes P G et al 1980 Macromolecules 131069

Dubruel P et al 2006 Surf. Sci. 6002562

Ivanovici S et al 2008 Macromolecules 411131

Kickelbick G 2003 Prog. Polym. Sci. 2883

Lahav M et al 2006 Nano Lett. 62166

Nam K et al 2010 Chem. Lett. 391164

Ohno K et al 2005 Macromolecules 382137

Paul D R and Robeson L M 2008 Polymer 493187

Sanchez C et al 2001 Chem. Mater. 133061 
Shirley D A 1972 Phys. Rev. B5 4709

Sudheendra L and Raju A R 1999 Bull. Mater. Sci. 221025

Tanaka Y et al 2007 J. Mater. Sci. Mater. Med. 18797

Tsujii Y et al 2006 Adv. Polym. Sci. 1971

Van Gils S et al 2004 Surf. Coat Technol. 185303

Weatherall I L 1992 Color Res. Appl. 17352
Wessling B 1997 Synth. Met. 851313

Xiao S -J et al 1998 Langmuir 145507

$\mathrm{Xu}$ W et al 2008 Small 6662

Yang Y et al 2006 Polymer 477374

Yoshikawa C et al 2006 Macromolecules 392284

Yoshikawa C et al 2010 Chem. Lett. 39142 